\title{
Analysis of Distributed Transmit Diversity with Outdated Diversity Weights
}

\author{
Sahar Amini, Kwonhue Choi, and Dongjun Na \\ Department of Information and Communication Engineering, Yeungnam University, Gyeongsan 712-749, Republic of Korea \\ Correspondence should be addressed to Kwonhue Choi; gonew@yu.ac.kr
}

Received 18 June 2017; Revised 22 August 2017; Accepted 20 September 2017; Published 26 October 2017

Academic Editor: Jose F. Monserrat

Copyright (C) 2017 Sahar Amini et al. This is an open access article distributed under the Creative Commons Attribution License, which permits unrestricted use, distribution, and reproduction in any medium, provided the original work is properly cited.

\begin{abstract}
Distributed transmit diversity (DTD) technique that combines cooperative communications and diversity techniques is a suitable solution in 5th-generation (5G) systems. In this paper, we investigate the effect of receiver phase compensation (RPC) on the performance of DTD. We introduce new expressions for the average error rate of DTD in the presence of RPC. The derived expressions are useful for a large number of modulation schemes. We obtain further insights by comparing the RPC effect on DTD with different spatially correlated collocated transmit diversity (CTD). The new observations include the following: (1) In the case of feedback delay, the phase compensation (PC) is required on the receiver side in addition to the transmitter side. (2) In DTD with RPC, the system performance is improved by increasing the differences between the channel gain variances. However, this is the opposite of the case of DTD without RPC. (3) The correlated CTD is more sensitive to RPC than DTD. This sensitivity increases by enhancing the correlation between transmit (TX) antennas. (4) In the case where there is no delay for CTD or DTD, RPC does not affect the system's performance.
\end{abstract}

\section{Introduction}

In the literature, transmit diversity (TD) systems are classified into distributed and collocated systems, which are referred to as distributed TD (DTD) and collocated TD (CTD), respectively. In CTD, multiple transmit (TX) antennas are located on a single transmitter. On the other hand, in DTD, these antennas are far apart and have different slow fading factors. Hence, in DTD, there is another diversity called macrodiversity, which is not available in CTD [1-5].

In cellular systems with DTD, user equipment (UE) is connected to individual antennas at various base stations (BSs). On the other hand, in cellular systems with CTD, the BS that has the highest mean channel gain (MCG) is connected to the considered UE. In the relevant literature, MCG is referred to as a large-scale fading factor, channel gain variance, or long-term fading factor. Unlike the DTD, where the MCGs are different among the connected TX antennas, all the TX antennas in CTD have the highest MCGs as the TX antennas are collocated, but there is a spatial correlation among the collocated TX antennas $[6,7]$.
Because of the realization of signal-to-noise ratio (SNR) maximization and coherent CTD and DTD, receiver-side channel state information (CSI) is needed in the transmitter. This is prepared by the CSI feedback from the receiver side to the transmitter side $[1-6,8]$. The practical performance of CTD and DTD suffers from several kinds of imperfections in CSI such as the feedback loop delay. Therefore, in actual systems, the available CSI at the transmitter and that at the channel do not exactly match [6]. Consequently, even though the channel measurement is perfect in the receiver, the outdated TX diversity weighting in the transmitter side makes the arriving signals from TX antennas be imperfectly (noncoherently) combined in front of the receive (RX) antenna due to the unknown channel variation during the delay time. This phenomenon causes the undesirable term in the received signal. The effects of the outdated CSI to the RX side diversity and the TX side diversity have been already reported in [9], [10], and [11], respectively. However, their system models are limited to the case of nondistributed (clustered) antennas. 


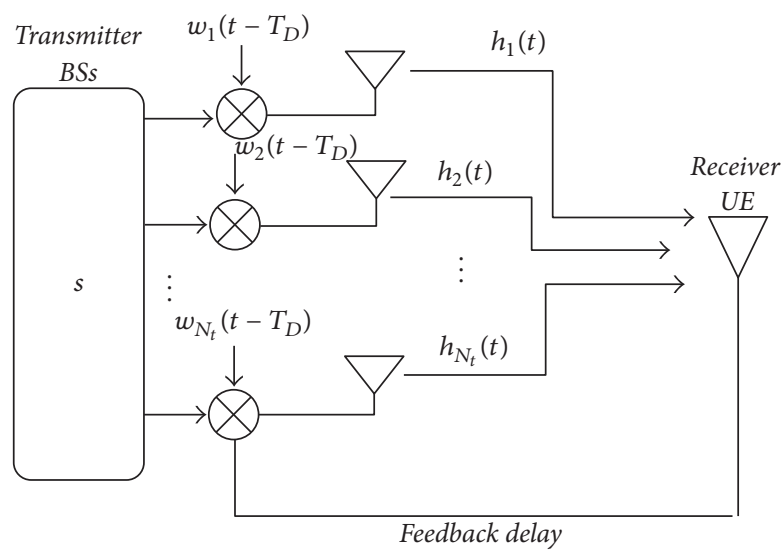

(a) Model of DTD and CTD with feedback delay

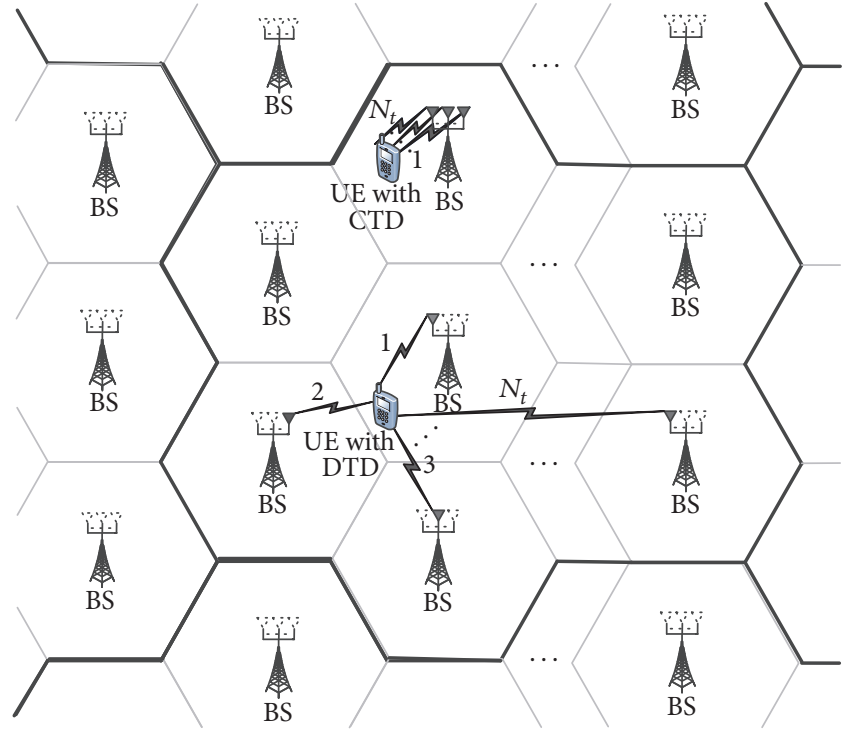

(b) Model of DTD and CTD in a cell

Figure 1: System model.

As mentioned above, the feedback delay in TX diversity systems incurs undesirable self-noise term in the received signal. This term disturbs the magnitude and the phase of the combined signal component in front of the RX antenna and thus degrades the performance of CTD and DTD systems. Therefore, to improve the system's performance, the compensation (derotation) for the disturbed phase is needed on the receiver side before detecting the signal. This compensation is to referred as RPC (receiver phase compensation) in this paper. Owing to price or space limitations, the RPC is sometimes excluded, for example, a very low complexity receiver such as machine-type communication (MTC) terminals, sensors, or Internet of Things (IoT) devices, which enables the receiver side to focus only on demodulation, while the transmitter side consists of all the preprocessing, such as precompensation of the phase rotation by the channel, by using CSI feedback from the receiver. At present, the joint effect of limited-rates and outdated feedback has been investigated in CTD systems without RPC $[12,13]$. The exact outage probability, ergodic capacity, and the average error rate performance of CTD in spatially correlated multipleinput single-output (MISO) systems with feedback delay and channel measurement error have been analyzed in [6]. In our previous work [14], we studied the performance of DTD with delay in the absence of RPC. However, the performance degradation by excluding RPC in DTD systems has not been investigated compared to DTD systems in the presence of RPC. Furthermore, the effect of RPC on DTD compared to CTD has not been established by the existing work.

In this paper, we investigate the effect of RPC on the DTD for the case when the transmit diversity weights are outdated due to feedback delay. By exploiting an accurate method for modeling the feedback delay [10, 11], we derive a theoretical average error rate expression of DTD with and without RPC. In addition, we analyze the effect of RPC on DTD and compare it with the correlated CTD. Both analytical and simulation studies have been carried out to show the effectiveness of the RPC on DTD and it is shown that RPC is crucial in the presence of feedback delay. Also, the results show that, in DTD with RPC, as the differences between the slow-fading factors increase, (e.g., as the UE gets close to one of the BSs), the performance improves, whereas the DTD without RPC shows the opposite trends. Besides, the correlated CTD is more sensitive than DTD to the RPC. This sensitivity increases by enhancing the correlation between TX antennas.

The paper is organized as follows. In Section 2, we present the channel and system models used in our study. Then, in Section 3, we analyze the average error rates for the DTD systems in the presence and absence of RPC as well as in the no-delay case. In Section 4, we provide numerical and simulation results to show the effect of RPC on DTD. In this section, we also compare the DTD performance with that of the correlated CTD. Finally, we conclude this paper in Section 5.

\section{System Model}

We consider a MISO system with $N_{t}$ distributed TX antennas for TD and one receiver antenna at each UE over a Rayleigh fading channel (Figure 1(a)). In correlated CTD systems, the UE connects to the strongest BS in terms of MCG, and the $N_{t}$ collocated TX antennas of the chosen BS are activated for TD to the UE. On the other hand, in DTD systems, the $N_{t}$ strongest BSs in terms of MCGs are selected first and then one antenna per BS, that is, $N_{t}$ distributed antennas in total, are selected (Figure 1(b)). As all antennas belonging to the same 
BS have the identical MCGs and their fast-fading factors are modeled to have the identical statistics, any one among them can be selected as an element of the distributed antennas to achieve the identical error rate performance.

In this model, the TD antennas are shared between the connected multiple UEs. The signals to the UEs are multiplexed in the code/time or frequency domains, as done in orthogonal frequency-division multiple access (OFDMA) or code-division multiple access (CDMA) and not in the spatial domain, as in multiuser (MU) multiple-input multipleoutput (MIMO). In this study, the use of TD is to improve the link quality and not for multiplexing in the spatial domain. The same usages of TD in OFDMA and CDMA have been studied in [15-17] and [18-20], respectively.

Common to collocated and distributed TDs, the received signal in each UE is calculated as $[6,10,11]$

$$
y=\mathbf{w}^{H} \mathbf{h} s+n,
$$

where $\mathbf{w}$ is the TD weighting factor, $(\cdot)^{H}$ denotes the conjugate transpose, and $s$ is the common transmitted symbol to $N_{t}$ TX antennas with $E\left\{|s|^{2}\right\}=E_{s}$. The channel vector is given by $\mathbf{h}=\left[h_{1}, h_{2}, \ldots, h_{N_{t}}\right]^{T}$, where the term $h_{l}$ is the overall channel fading coefficient of the $l$ th TX antenna and $(\cdot)^{T}$ denotes the transpose. This channel model is equivalent to the composite Rayleigh fading model, which can be expressed as follows [3]:

$$
h_{l}=f_{l} \sqrt{g_{l}} .
$$

The first factor $f_{l}$ is the fast and small-scale fading factor with zero mean and unit variance complex Gaussian distribution. The second factor $g_{l}$ is the slow and large-scale fading factor determined by shadowing or path loss. The large-scale fading factors are changing very slowly, that is, quasi-static. Thus, we analyze the system performance for the various cases when the large-scale fading factors are differently given. This allows us to treat the large-scale fading factors as the temporarily deterministic variables in the derivations. In this model, $g_{l}$ can be treated as the power of the overall channel fading factor $h_{l}$ averaged over $f_{l}$. Thus, we refer to $g_{l}$ as the MCG of the $l$ th TX antenna to a specific UE in a cell. The term $n$ is the zero mean complex additive white Gaussian noise (AWGN) with variance $N_{0} / 2$. As shown in $[3,17]$, the inter-UE interference is excluded in (1) by assuming that the UEs are multiplexed in the orthogonal resources (such as code, time, or frequency domain). According to the principles of maximal ratio transmission, the optimal TD weighting vector is defined as follows:

$$
\mathbf{w}=\frac{\mathbf{h}}{\sqrt{\sum_{l=1}^{N_{t}}\left|h_{l}\right|^{2}}}=\frac{\mathbf{h}}{\|\mathbf{h}\|_{F}},
$$

where $\mathbf{h}$ is the channel vector and $\|\cdot\|_{F}$ denotes the Frobenius norm $[3-6,10,11]$.

In the case involving delay, the term $h_{l}\left(t-T_{D}\right)$ is the $l$ th TX antenna's outdated instantaneous channel fading factor measurement, which is fed back to the transmitter and used at time $t$. Therefore, $h_{l}(t)$ denotes the $l$ th TX antenna's current instantaneous channel fading factor. Note that $\mathbf{w}$ is calculated from the outdated instantaneous channel fading factor $\mathbf{h}(t-$ $T_{D}$ ) instead of the current instantaneous channel fading factor $\mathbf{h}(t)$. We assume that the variance of the channel fading factor satisfies

$$
\sigma_{h_{l}(t)}^{2}=\sigma_{h_{l}\left(t-T_{D}\right)}^{2}=g_{l} .
$$

This assumption is acceptable because, in practical systems, the coherence time of the MCG, which defines the variance of the overall channel fading factor, is much higher than the feedback delay. The correlation coefficient between $h_{l}(t)$ and $h_{l}\left(t-T_{D}\right)$ is determined by Jake's model or Clarke's fading spectrum as $\rho_{d, l}=J_{0}\left(2 \pi f_{D, l} T_{D}\right)$, where $f_{D, l}$ and $T_{D}$ are the Doppler frequency of the $l_{\text {th }}$ TX antenna and feedback loop delay, respectively, and $J_{0}(x)$ denotes the first-kind zerothorder Bessel function. As each signal path from the distributed antennas experiences different relative velocity and thus different Doppler frequency, the correlation coefficients $\rho_{d, l}$ are set nonidentically for different $l$. The correlation model between $h_{l}\left(t-T_{D}\right)$ and $h_{l}(t)$, which satisfies Clarke's model and (4), is defined as follows $[2,5,6,10,11]$ :

$$
\begin{aligned}
h_{l}(t)=\rho_{d, l} h_{l}\left(t-T_{D}\right)+\sqrt{1-\rho_{d, l}^{2}} e_{l}(t), & \\
& \quad l=1, \ldots, N_{t},
\end{aligned}
$$

where $e_{l}$ is the independent complex Gaussian random variables with zero mean and variance $g_{l}$.

\section{Error Rate Analysis for DTD Systems}

In this section, we derive the average error rate of DTD systems with and without RPC, respectively, using the statistical characterization of the SNR.

3.1. Average Error Rate in the Presence of RPC. With a feedback delay $T_{D}$, the received SNR of TD systems at time $t$ in the presence of RPC is defined as follows:

$$
\gamma\left(t \mid T_{d}\right)=\left|\mathbf{w}^{H}\left(t-T_{d}\right) \mathbf{h}(t)\right|^{2} \bar{\gamma}_{s},
$$

where $\bar{\gamma}_{s}=E_{s} / N_{0}$ is the average symbol SNR from one of the TX antennas when its variance of the overall channel fading factor is equal to one. Note that as we are considering the case when the channel measurement is perfect but there is a feedback delay $T_{D}$, the weighting vector $\left|\mathbf{w}^{H}(t)\right|$ in (1) is replaced by its outdated version $\left|\mathbf{w}^{H}\left(t-T_{D}\right)\right|$ in (6), which is equal to $\left|\mathbf{h}^{H}\left(t-T_{D}\right)\right| /\left\|\mathbf{h}\left(t-T_{D}\right)\right\|_{F}$ from (3).

By substituting (5) and $\left|\mathbf{w}^{H}\left(t-T_{D}\right)\right|\left(=\left|\mathbf{h}^{H}\left(t-T_{D}\right)\right| / \| \mathbf{h}(t-\right.$ $\left.T_{D}\right) \|_{F}$ ) into (6), the received SNR is rewritten as follows:

$$
\begin{aligned}
& \gamma\left(t \mid T_{d}\right)=\mid \frac{1}{\left\|\mathbf{h}\left(t-T_{d}\right)\right\|_{F}}\left(\sum_{l=1}^{N_{t}} \rho_{d, 1}\left|h_{l}\left(t-T_{d}\right)\right|^{2}\right. \\
& \left.+\mathbf{h}\left(t-T_{d}\right)^{H} \mathbf{e}_{d}(t)\right)\left.\right|^{2} \bar{\gamma}_{s},
\end{aligned}
$$

where $\mathbf{e}_{d}(t)=\left[\sqrt{1-\rho_{d, 1}^{2}} e_{1}, \sqrt{1-\rho_{d, 2}^{2}} e_{2}, \ldots, \sqrt{1-\rho_{d, N_{t}}^{2}} e_{N_{t}}\right]$. 
In general, the delayed CSI feedback causes performance degradation. In this subsection, we present a new closedform expression for the moment generating function (MGF) of SNR in DTD with RPC systems. In order to derive the MGF of the SNR, the conditional MGF, $M_{\gamma\left(t \mid T_{D}\right)}(s \mid \mathbf{h})$, is first calculated by considering that the received SNR has a noncentral Gaussian quadratic distribution conditioned on $\mathbf{h}\left(t-T_{D}\right)$. Then, $M_{\gamma\left(t \mid T_{D}\right)}(s \mid \mathbf{h})$ is averaged over the outdated channel to calculate the average MGF $M_{\gamma\left(t \mid T_{D}\right)}(s)$ [11].

The received SNR conditioned on the feedback loop delay can be calculated in a complex Gaussian quadratic form as follows:

$$
\gamma\left(t \mid T_{D}\right)=x^{*} \bar{\gamma}_{s} x
$$

where

$$
\begin{aligned}
x= & \frac{1}{\left\|\mathbf{h}\left(t-T_{d}\right)\right\|_{F}}\left(\sum_{l=1}^{N_{t}} \rho_{d, 1}\left|h_{l}\left(t-T_{d}\right)\right|^{2}\right. \\
& \left.+\mathbf{h}\left(t-T_{d}\right)^{H} \mathbf{e}_{d}(t)\right)
\end{aligned}
$$

it follows that

$$
\begin{aligned}
x \sim C N\left(\frac{\sum_{l=1}^{N_{t}}\left(\rho_{d, 1}\left|h_{l}\left(t-T_{d}\right)\right|^{2}\right)}{\left\|\mathbf{h}\left(t-T_{d}\right)\right\|_{F}},\right. \\
\frac{\sum_{l=1}^{N_{t}}\left(\left|h_{l}\left(t-T_{d}\right)\right|^{2}\left(1-\rho_{d, l}^{2}\right) g_{l}\right)}{\left\|\mathbf{h}\left(t-T_{d}\right)\right\|_{F}^{2}} \\
\left.=\frac{\sum_{l=1}^{N_{t}}\left(\left|f_{l}\right|^{2} g_{l}^{2}\left(1-\rho_{d, l}^{2}\right)\right)}{\sum_{l=1}^{N_{t}}\left(\left|f_{l}\right|^{2} g_{l}\right)}\right) .
\end{aligned}
$$

We can treat $x$ in (10) as a Gaussian variable only when the random variables $h_{1}, h_{2}, h_{3}, \ldots, h_{N_{T}}$ are given (conditioned). The exact statistics of unconditioned $x$ are hard to characterize and thus the further derivation with the exact PDF of $x$ is cumbersome. As an alternative technique, we employ the average error rate derivation technique in [11]. To apply this technique, the variance of $x$ in (10) should be deterministic. However, (10) is a random variable, since the variance term has the random variables $f_{1}, f_{2}, \ldots, f_{N_{t}}$ as mentioned above. In order to ameliorate this problem, we replace $\left|f_{l}\right|^{2}$ by its mean, that is, 1 . Then, (10) can be rewritten as

$$
\begin{gathered}
x \sim \operatorname{CN}\left(\frac{\sum_{l=1}^{N_{t}}\left(\rho_{d, 1}\left|h_{l}\left(t-T_{d}\right)\right|^{2}\right)}{\left\|\mathbf{h}\left(t-T_{d}\right)\right\|_{F}},\right. \\
\left.\frac{\sum_{l=1}^{N_{t}}\left(g_{l}^{2}\left(1-\rho_{d, l}^{2}\right)\right)}{\kappa \sum_{l=1}^{N_{t}} g_{l}^{2}}\right),
\end{gathered}
$$

where $\kappa$ is a normalization factor given as $\sqrt{\sum_{l=1}^{N_{t}} g_{l} / N_{t}}$.

Note that although the overall channel fading factors $\left(h_{1}\left(t-T_{D}\right), h_{2}\left(t-T_{D}\right), \ldots, h_{N_{t}}\left(t-T_{D}\right)\right)$ are conditioned, $x$ in (11) is complex Gaussian and thus $\gamma$ in (8) has a Chisquared distribution. This is because the unknown channel variation factor during the feedback delay time, that is, $\mathbf{e}_{d}(t)$ in (9), is modeled as Gaussian. By using the result of the Gaussian quadratic distributions [21] and ([11],(8)), we obtain the conditional MGF of the SNR as follows:

$$
\begin{aligned}
& M_{\gamma\left(t \mid T_{D}\right)}(s \mid h) \\
& \quad=\exp \left\{\frac{\left|\rho_{d, 1}\right|^{2}\left\|h\left(t-T_{d}\right)\right\|_{F}^{2}}{s^{-1} \bar{\gamma}_{s}^{-1}-\sqrt{N_{t}}\left(\sum_{l=1}^{N_{t}} g_{l}\right)^{-1.5} \sum_{l=1}^{N_{t}}\left(g_{l}^{2}\left(1-\rho_{d, l}^{2}\right)\right)}\right\} \\
& \quad \times\left(1-s \bar{\gamma}_{s}\left(\frac{\sqrt{N_{t}} \sum_{l=1}^{N_{t}}\left(g_{l}^{2}\left(1-\rho_{d, l}^{2}\right)\right)}{\left(\sum_{l=1}^{N_{t}} g_{l}\right)^{1.5}}\right)\right) .
\end{aligned}
$$

According to the similar procedure of deriving a closedform PDF expression in the received SNR as ([11], (15)), the pdf expression for DTD can be calculated as

$$
\begin{aligned}
f_{\gamma\left(t \mid T_{D}\right)}(x) & \frac{1}{\left(\bar{\gamma}_{s}\left(\alpha \sum_{l=1}^{N_{t}}\left(g_{l}^{2}\left(1-\rho_{d, l}^{2}\right)\right)+\left|\rho_{d, 1}\right|^{2} \beta\right)\right)^{N_{t}-1}} \\
& \cdot \sum_{n=0}^{N_{t}-1} \frac{\left(\begin{array}{c}
N_{t}-1 \\
n
\end{array}\right)}{\left(N_{t}-1-n\right) !} \\
& \cdot\left(1-\frac{\alpha \sum_{l=1}^{N_{t}}\left(g_{l}^{2}\left(1-\rho_{d, l}^{2}\right)\right)}{\alpha \sum_{l=1}^{N_{t}}\left(g_{l}^{2}\left(1-\rho_{d, l}^{2}\right)\right)+\beta\left|\rho_{d, 1}\right|^{2}}\right)^{N_{t}-1-n} \\
& \times\left(\alpha \bar{\gamma}_{s}^{N_{t}}\left(g_{l=1}^{2}\left(1-\rho_{d, l}^{2}\right)\right)\right)^{n} x^{N_{t}-1-n} \\
& \cdot \exp \left\{\frac{-x}{\bar{\gamma}_{s}\left(\alpha \sum_{l=1}^{N_{t}}\left(g_{l}^{2}\left(1-\rho_{d, l}^{2}\right)\right)+\left|\rho_{d, 1}\right|^{2} \beta\right)}\right\},
\end{aligned}
$$

where $\alpha=\sqrt{N_{t}}\left(\sum_{l=1}^{N_{t}} g_{l}\right)^{-1.5}$ and $\beta=\sqrt{N_{t}}\left(\sum_{l=1}^{N_{t}} g_{l}^{2}\right) \times$ $\left(\sum_{l=1}^{N_{t}} g_{l}\right)^{-1.5}$. Then, by using the asymptotic average error rate expression ([22], Proposition (1)) and following the average error rate derivation of CTD in ([10], (23) and (24)), we derive the simple, compact, and accurate average error rate equation for DTD systems in the presence of RPC as follows. (It is written $g(x)=o(f(x))$ as $x \rightarrow x_{0}$, if $\lim _{x \rightarrow x_{0}}(g(x) / f(x))=$ 0.)

$$
P_{\mathrm{wRPC}}=\frac{a_{s}(1 / N t) \sum_{l=1}^{N_{t}} \prod_{k \neq l}\left(1-\rho_{d, k}^{2}\right)}{2 b_{s} \bar{\gamma}_{s} \beta}+o\left(\beta \bar{\gamma}_{s}^{-2}\right) .
$$

Two constant parameters, $a_{s}$ and $b_{s}$, depend on modulation schemes, and $\beta$ is defined in (13). Equation (14) is rather contrary to an insight that the diversity order should be equal to $N_{t}$. However, this insight is true when there is no feedback delay. If the feedback delay increases and thus the self-interference term due to outdated diversity weighting becomes significant, then the higher-order diversity term $o\left(\beta \bar{\gamma}_{s}^{-2}\right)$ is getting negligible compared to the first-order 
diversity term. This asymptotically decreases the diversity order back to 1 in the nominally high SNR range [10]. This phenomenon is more magnified in DTD compared to CTD because the differences of MCGs among the diversity TX antennas by themselves reduce the diversity gain.

3.2. Average Error Rate in the Absence of RPC. By substituting (3) and (5) into (1), the received signal of the TD systems at time $t$ is given as follows:

$$
\begin{aligned}
y(t)= & \sum_{l=1}^{N_{t}} w_{l}^{*}\left(t-T_{D}\right) h_{l}(t) s+n(t) \\
= & \sum_{l=1}^{N_{t}} \frac{h_{l}^{*}\left(t-T_{D}\right)}{\sqrt{\sum_{i=1}^{N_{t}}\left|h_{i}\left(t-T_{D}\right)\right|^{2}}} h_{l}(t) s+n(t) \\
= & \frac{\sum_{l=1}^{N_{t}}\left(\rho_{d, l}\left|h_{l}\left(t-T_{D}\right)\right|^{2}\right) s}{\sqrt{\sum_{l=1}^{N_{t}}\left|h_{l}\left(t-T_{D}\right)\right|^{2}}} \\
& +\frac{\sum_{l=1}^{N_{t}} h_{l}^{*}\left(t-T_{D}\right) \sqrt{1-\rho_{d, l}^{2}} e_{l}(t)}{\sqrt{\sum_{i=1}^{N_{t}}\left|h_{l}\left(t-T_{D}\right)\right|^{2}}} s+n(t),
\end{aligned}
$$

where the term $w_{l}$ denotes the TD weighting factor from $l_{\text {th }}$ TX antennas and the variance of the noise is equal to $N_{0} / 2$.

The second term in (15) comes from the mismatch between the outdated diversity weights in TX side and the actual channel fading factor. In this subsection, we are considering the systems with very low complexity receivers such as MTC terminals, sensors, or IoT devices. Thus, in order to simplify the receiver processing bearing the performance loss as done in $[12,13]$, we skip the RPC, that is, the compensation (derotation) for the phase disturbance by the second term in (15), and just take the received signal as is for detection. Then, as the term $e_{l}(t)$ is modeled as complex Gaussian in (5), the second term in (15) is just treated as an unknown interference in the received signal. This is the essential difference from the case in Section 3.1. Thus, the received signal is rewritten as

$$
y(t)=\frac{\sum_{l=1}^{N_{t}}\left(\rho_{d, l}\left|h_{l}\left(t-T_{D}\right)\right|^{2}\right)}{\left\|\mathbf{h}\left(t-T_{D}\right)\right\|_{F}} s+n_{\text {total }}(t),
$$

where $n_{\text {total }}(t)=\left(\sum_{l=1}^{N_{t}} h_{l}^{*}\left(t-T_{D}\right) \sqrt{1-\rho_{d, l}^{2}} e_{l}(t) / \| \mathbf{h}(t-\right.$ $\left.\left.T_{D}\right) \|_{F}\right) s+n(t)$. From the fact that the overall channel fading factor $h_{l}(t)$ is statistically identical irrespective of the time index $t$, we can detach the time variable. Then, we have

$$
y=\frac{\sum_{l=1}^{N_{t}}\left(\rho_{d, l}\left|h_{l}\right|^{2}\right)}{\|\mathbf{h}\|_{F}} s+n_{\text {total }}
$$

where $n_{\text {total }}=\left(\sum_{l=1}^{N_{t}} h_{l}^{*} \sqrt{1-\rho_{d, l}^{2}} e_{l}(t) /\|\mathbf{h}\|_{F}\right) s+n$. From (17), the overall SNR can be calculated as follows:

$$
\mathrm{SNR}=\frac{E\left\{\left|\sum_{l=1}^{N_{t}}\left(\rho_{d, l}\left|h_{l}\right|^{2}\right)\right|^{2}\right\} E_{s}}{\|\mathbf{h}\|_{F}^{2} E\left\{\left|n_{\text {total }}\right|^{2}\right\}},
$$

where $E\left\{\left|n_{\text {total }}\right|^{2}\right\}=N_{0} / 2+E_{s}\left(\sum_{l=1}^{N_{t}}\left(1-\rho_{d, l}^{2}\right)\left|h_{l}\right|^{2} /\|\mathbf{h}\|_{F}{ }^{2}\right)$. It is obvious that, in the overall SNR, there is no term regarding the compensation of the phase difference between outdated and current channels.

Using the SNR statistical characterization, the average error rate of DTD without RPC is calculated in our previous work [14] as

$$
\begin{aligned}
P_{\mathrm{woRPC}}= & \sum_{l=1}^{t} \sum_{i=1, i \neq l}^{t} a_{s} S_{l} g_{l}^{t-2} B_{i, l}^{N_{t}-3} Q_{i, l}^{-1} \\
& \cdot\left(\prod_{m=1, m \neq l, m \neq i}^{N_{t}}\left(B_{i, l}-B_{m, l}\right)^{-1}\right) \\
& \cdot\left(\beta_{l} \sqrt{\frac{b_{s} \beta_{l}}{b_{s} \beta_{l}+2 q^{2}}}-\beta_{l}+D\right),
\end{aligned}
$$

where $S_{l}=\prod_{i=1, i \neq l}^{N_{t}}\left(g_{l}-g_{i}\right)^{-1}, B_{i, l}=\left(g_{l} \beta_{i}-g_{i} \beta_{l}\right) /\left(g_{l}-g_{i}\right)$, $\beta_{l}=\left(g_{l} N_{0} / 2+E_{s} \alpha_{l}\right) / b, \alpha_{l}=\left(1-\rho_{d, l}{ }^{2}\right) g_{l}^{2}, Q_{i, l}=\left(B_{i, l}-\beta_{l}\right) / g_{l} B_{i, l}$, and $q_{l}=\beta_{l} / g_{l}$ and $D$ is given as

$$
\begin{aligned}
D= & -B_{i, l} \sqrt{\frac{b_{s} \beta_{l}}{b_{s} \beta_{l}+2 q_{l}^{2}}}+B_{i, l} \\
& -\frac{Q_{i, l} B_{i, l}}{\sqrt{2}} \frac{2 q_{l}+Q_{i, l} B_{i, l}}{2 b_{s}-Q_{i, l}^{2} B_{i, l}} \sqrt{\frac{2 B_{i, l} b_{s}}{B_{i, l} b_{s}+2 q_{l}^{2}+2 B_{i, l} Q_{i, l} q_{l}}} \\
& +\frac{Q_{i, l}^{2} B_{i, l}^{2}}{2 b_{s}-Q_{i, l}^{2} B_{i, l}} .
\end{aligned}
$$

3.3. Average Error Rate in No-Delay DTD. Let us now consider the case of DTD in an ideal environment without delay. Because we omit the error term of the delay in ideal TD systems, RPC does not affect their performance. The average error rate formula for the no-delay DTD system is calculated in our previous work as [4]

$$
P_{\text {NoDelay }}=\frac{a_{s}}{2} \sum_{l=1}^{N_{t}} \varphi_{l}\left[1-\sqrt{\frac{g_{l} b_{s} \gamma}{2+g_{l} b_{s} \gamma}}\right] \text {, }
$$

where the SNR $\gamma=E_{b} / N_{0}$ ( $E_{b}$ is the bit energy) and $\varphi_{l} \triangleq \prod_{i \neq l}\left(g_{l} /\left(g_{l}-g_{i}\right)\right)$. As mentioned before, two constant parameters, $a_{s}$ and $b_{s}$, depend on the modulation scheme.

\section{Simulation and Numerical Results}

In this section, we perform Monte Carlo simulations to validate the analytical results derived in previous subsections, and we investigate the effect of RPC and the feedback loop delay on the performance of DTD and CTD systems. As previously mentioned, the channel model is considered as a composite Rayleigh fading that consists of small-scale fading and MCG (shadowing or path loss). Unless otherwise specified, for simplicity, we assume that $g_{2}>g_{1}=0 \mathrm{~dB}, \bar{\gamma}_{s}=\gamma$, and $N_{t}=2$, which is extendable to other numbers of TX antennas. Furthermore, from (14), (19), and (21), it is clear that, by 


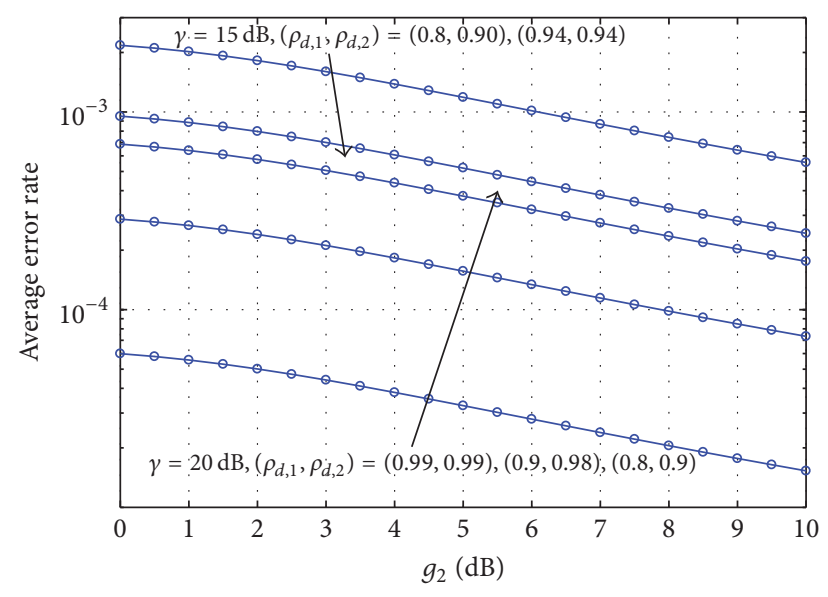

- $P_{\text {wRPC }}$ by equation (14)

- Monte Carlo simulation

FIGURE 2: Simulated and analytical average error rate of DTD in the presence of RPC with various delay values and parameters: $\gamma=$ $15,20 \mathrm{~dB}, g_{1}=0 \mathrm{~dB}$, and $N_{t}=2$.

changing the scaling factors, $a_{s}$ and $b_{s}$, versus the average errors rates, we obtain different modulation conditions. Thus, the conclusion of each modulation is applicable to other modulation schemes. In this paper, without loss of generality, the theoretical results are restricted to the binary phase-shift keying (BPSK) modulation scheme $\left(a_{s}=1\right.$ and $\left.b_{s}=2\right)$.

Figure 2 shows the performance of DTD in the presence of RPC for the various correlation factor pairs (=the various feedback delays) and the various MCG pairs. It is shown that the theoretical results, which are plotted using (14), agree with the simulations in all MCG ranges, which verifies the correctness of our analysis. From those curves, it is obvious that as the feedback delay increases, that is, $\rho_{d, l}$ decreases, the performance degrades. On the other hand, as the MCG values $\left(g_{2}\right)$ of the TX antennas increase, the performance improves.

Figure 3 presents the performance of DTD in the absence of RPC for the various correlation factor pairs (=the various feedback delays) and the various MCG pairs. For reference, the cases of no delay, that is, $\rho_{d, 1}=\rho_{d, 2}=1$, are included. The results show that as the feedback delay slightly increases, that is, $\rho_{d, l}$ slightly decreases from 1 , the performance significantly degrades. It is remarkable that, unlike DTD with RPC, as $g_{2}$ increases, that is, as the gap between the MCGs from the BSs increases, the performance of DTD without RPC degrades. This is because as the MCG difference increases, the phase disturbing self-interference term proportionally increases due to no RPC and, moreover, the diversity gain decreases as well [2]. Although in DTD with RPC, when the MCG difference increases, we lose the diversity gain as well, the RPC compensates the phase error disturbed by the selfinterference term. Therefore, in cases involving DTD with RPC, as $g_{2}$ increases, the performance improves. Meanwhile, we confirm that the simulated average error rate of DTD systems without RPC and no-delay cases agree exactly with the analytical values calculated by (19) and (21), respectively.

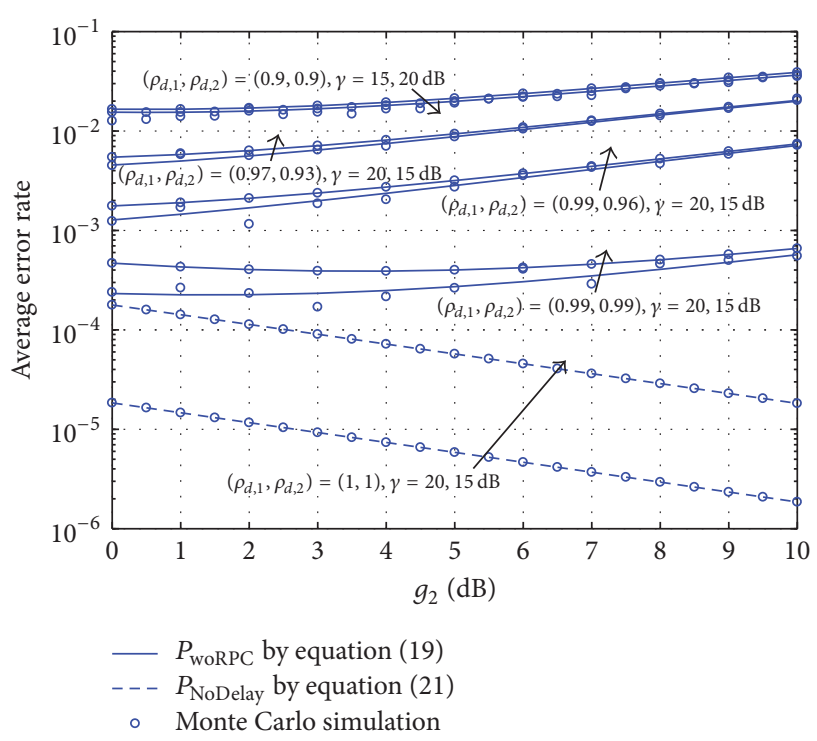

FIGURE 3: Simulated and analytical average error rate of DTD in the absence of RPC [14] and no-delay [4] cases with $\gamma=15,20 \mathrm{~dB}, g_{1}=$ $0 \mathrm{~dB}$, and $N_{t}=2$.

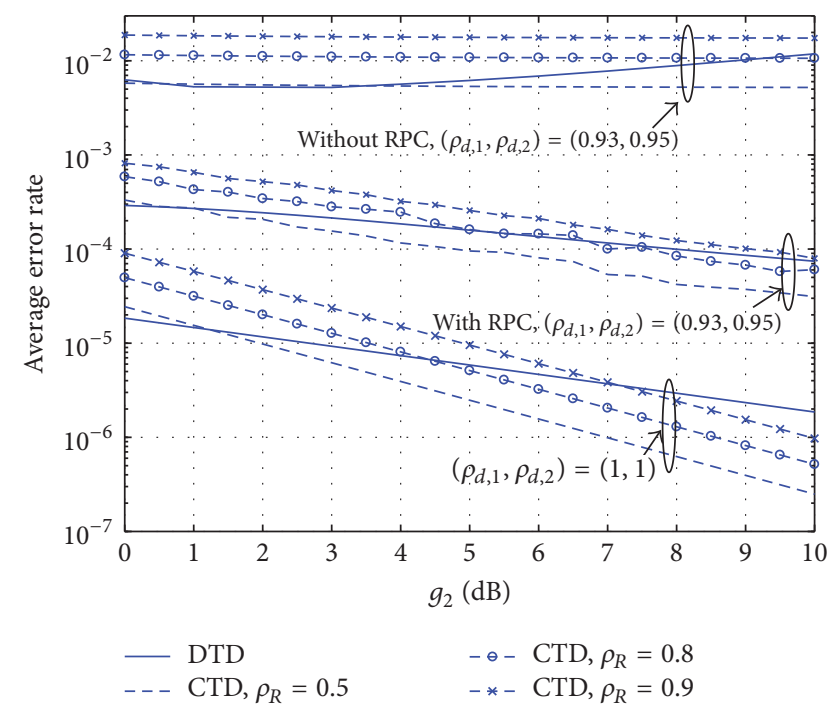

FIgURE 4: Average error rate comparison of DTD and correlated CTD versus MCG $\left(g_{2}\right)$ with $N_{t}=2, g_{1}=0 \mathrm{~dB}$, and $\gamma=20 \mathrm{~dB}$.

We observe that the exclusion of RPC is critical in largedelay (=small $\left.\rho_{d, l} \mathrm{~s}\right)$ cases, and the performance is in the unacceptable range. This implies in turn that only in the very small-delay cases can the RPC be skipped with relatively less performance loss.

Figure 4 evaluates the effect of RPC on the correlated CTD and DTD systems for the various correlation factor pairs (=the various feedback delays), the various MCG pairs, and the various spatial correlation coefficient (SCC) values of 


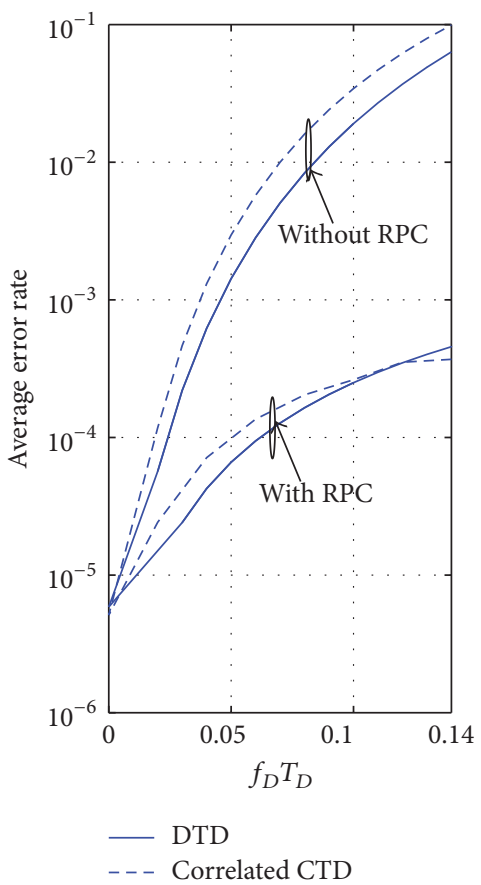

(a) The various Doppler frequency and delay product with $\rho_{R}=0.8$

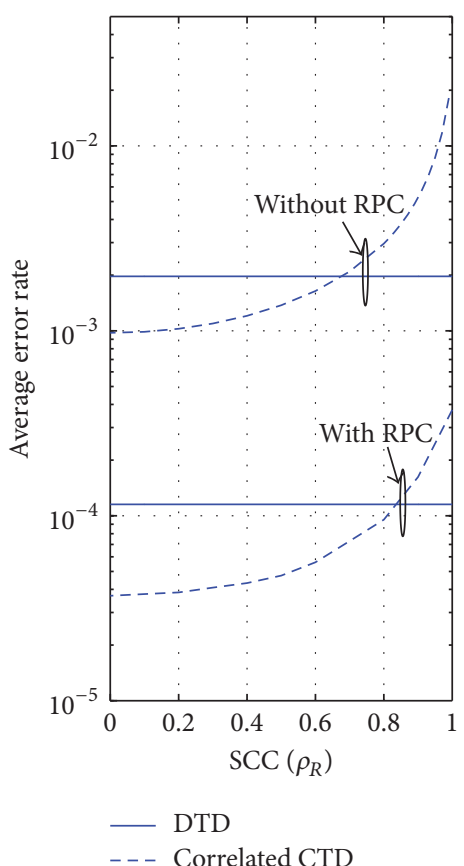

(b) The various $\operatorname{SCC} \rho_{R}$ with $\left(f_{D 1} T_{D}\right.$, $\left.f_{D 2} T_{D}\right)=(0.08,0.05)$

FIGURE 5: Average error rate comparison of DTD and correlated CTD in detail, according to RPC with $N_{t}=2,\left[g_{1}, g_{2}\right]=[0,5] \mathrm{dB}$, and $\gamma=20 \mathrm{~dB}$.

the clustered antennas. We consider the exponential channel correlation matrix for CTD as follows:

$$
R=\left(\begin{array}{cccc}
1 & \rho_{R} & \cdots & \rho_{R}{ }^{N_{t}-1} \\
\rho_{R} & 1 & \cdots & \rho_{R}{ }^{N_{t}-2} \\
\vdots & \vdots & \ddots & \vdots \\
\rho_{R}{ }^{N_{t}-1} & \rho_{R}{ }^{N_{t}-2} & \cdots & 1
\end{array}\right),
$$

where $0<\rho_{R}<1$ is the SCC between adjacent antennas. In this correlation model, a larger $\rho_{R}$ value implies a higher correlation between the TX antennas $[3,6]$. The results show that the performance of DTD is robust to the lack of RPC compared to the correlated CTD. This is due to the macrodiversity nature of DTD. This is especially true for the range of small $g_{2}$ values, where the MCG difference decreases and thus the diversity gain increases.

Finally, to obtain further insights, we analyze in detail the effect of RPC on DTD compared to the correlated CTD. Figure 5 compares the average error rates of the correlated CTD and DTD. In Figure 5(a), the average error rates are plotted as a function of feedback delay and Doppler frequency product while fixing SCC $\left(\rho_{R}\right)$. For simple presentation, we set the identical feedback delay and Doppler frequency product to each of distributed antennas without loss of generality. In Figure 5(b), the average error rates are plotted as a function of SCC $\left(\rho_{R}\right)$ while fixing feedback delay and Doppler frequency product. Figure 5(a) shows that as the feedback delay increases, both CTD and DTD get worse significantly and the degradation gets much more significant. Thus, RPC is critical in large-delay regions for both TD systems. Figure 5(b) shows that the correlated CTD is more sensitive to the RPC compared to the DTD. This sensitivity of the correlated CTD to RPC increases as the SCC between TX antennas increases, owing to the absence of the diversity. For example, when SCC $=0.8$ and $\left(f_{D, 1} T_{D}, f_{D, 2} T_{D}\right)=(0.08,0.05)$ in the presence of RPC, the preferred TD scheme is correlated CTD. However, in the absence of RPC, the preferred TD scheme is DTD.

\section{Conclusions}

In this paper, we investigated the impact of the feedback loop delay and RPC on the average error rate of DTD over Rayleigh fading channels. We presented the analytical error rate expressions and simulation results to show the effectiveness of the study based on DTD. The results show that not only the transmitter side PC (weighting factors) but also the receiver side PC is needed in the case of existing feedback delay. In the case where the minimized receiver side is preferred to the small improvements in the system performance, RPC is omitted in the small-size feedback loop delay. The results also present that as the difference between the MCGs increases (e.g., UE gets close to one of the BSs), the performance of DTD with RPC improves. However, this is opposite to the case without any RPC. We performed the comparison for the DTD performance with conventional correlated CTD, and we conclude that, in the case of existing 
feedback loop delay and excluding RPC, DTD is preferable over conventional correlated CTD, except for the cases where there are large differences between the MCGs. Finally, we conclude that, in the no-delay cases, RPC does not affect the performance of CTD and DTD systems.

\section{Conflicts of Interest}

The authors declare that there are no conflicts of interest regarding the publication of this paper.

\section{Acknowledgments}

This research was supported by Basic Science Research Program through the National Research Foundation (NRF) (2015R1D1A3A01015970) funded by the Ministry of Education, Republic of Korea.

\section{References}

[1] R. Mudumbai, D. R. Brown III, U. Madhow, and H. V. Poor, "Distributed transmit beamforming: challenges and recent progress," IEEE Communications Magazine, vol. 47, no. 2, pp. 102-110, 2009.

[2] K. Choi and H. Liu, "Power allocation for distributed transmit diversity with feedback loop delay," IEEE Transactions on Communications, vol. 59, no. 1, pp. 52-58, 2011.

[3] S. Amini and K. Choi, "Switching between co-located and distributed transmit diversity," IET Communications, vol. 10, no. 13, pp. 1614-1622, 2016.

[4] S. Amini, D.-J. Na, and K. Choi, "Performance comparison between distributed beamforming and clustered beamforming," in Proceedings of the 11th International Conference on Information Technology: New Generations (ITNG '14), pp. 174-179, IEEE, Las Vegas, Nev, USA, April 2014.

[5] S. Amini and K. Choi, "Effect of feedback delay and channel gain difference on distributed beamforming," in Proceedings of the 2011 International Conference on ICT Convergence, ICTC 2011, pp. 327-332, kor, September 2011.

[6] A. Firag, P. J. Smith, H. A. Suraweera, and A. Nallanathan, "Performance of beamforming in correlated MISO systems with estimation error and feedback delay," IEEE Transactions on Wireless Communications, vol. 10, no. 8, pp. 2592-2602, 2011.

[7] M. Kang and M.-S. Alouini, "Impact of correlation on the capacity of MIMO channels," in Proceedings of the IEEE International Conference on Communications (ICC '03), pp. 2623-2627, May 2003.

[8] A. Narula, M. J. Lopez, M. D. Trott, and G. W. Wornell, "Efficient use of side information in multiple-antenna data transmission over fading channels," IEEE Journal on Selected Areas in Communications, vol. 16, no. 8, pp. 1423-1436, 1998.

[9] P. Kumar and P. R. Sahu, "Analysis of M-PSK with MRC receiver over $\kappa-\mu$ fading channels with outdated CSI," IEEE Wireless Communications Letters, vol. 3, no. 6, pp. 557-560, 2014.

[10] H. A. Suraweera, T. A. Tsiftsis, G. K. Karagiannidis, and A. Nallanathan, "Effect of feedback delay on amplify-and-forward relay networks with beamforming," IEEE Transactions on Vehicular Technology, vol. 60, no. 3, pp. 1265-1271, 2011.

[11] Y. Ma, D. Zhang, A. Leith, and Z. Wang, "Error performance of transmit beamforming with delayed and limited feedback,"
IEEE Transactions on Wireless Communications, vol. 8, no. 3, pp. 1164-1170, 2009.

[12] J. W. Kang, M. Jang, S.-H. Kim, and D. I. Kim, "Outage analysis of multi-antenna rate adaptive systems with outdated feedback," IEEE Transactions on Wireless Communications, vol. 13, no. 10, pp. 5453-5466, 2014.

[13] D. A. Basnayaka, P. J. Smith, and P. A. Martin, "The effect of macrodiversity on the performance of maximal ratio combining in flat rayleigh fading," IEEE Transactions on Communications, vol. 61, no. 4, pp. 1384-1392, 2013.

[14] S. Amini, D.-J. Na, and K. Choi, "Effect of feedback delay and channel gain difference on distributed transmit diversity without receiver phase compensation," in Proceedings of the 2016 International Conference on Information and Communication Technology Convergence, ICTC 2016, pp. 1015-1019, October 2016.

[15] F. Rashid-Farrokhi, K. J. R. Liu, and L. Tassiulas, "Transmit beamforming and power control for cellular wireless systems," IEEE Journal on Selected Areas in Communications, vol. 16, no. 8, pp. 1437-1449, 1998.

[16] A. F. Naguib, A. Paulraj, and T. Kailath, "Capacity Improvement with Base-Station Antenna Arrays in Cellular CDMA," IEEE Transactions on Vehicular Technology, vol. 43, no. 3, pp. 691-698, 1994.

[17] Y. Zhou, F. Chin, Y.-C. Liang, and C.-C. Ko, "Performance comparison of transmit diversity and beamforming for the downlink of DS-CDMA system," IEEE Transactions on Wireless Communications, vol. 2, no. 2, pp. 320-334, 2003.

[18] M.-O. Pun, J. K. Kyeong, and H. V. Poor, "Opportunistic scheduling and beamforming for MIMO-OFDMA downlink systems with reduced feedback," in Proceedings of the IEEE International Conference on Communications, ICC 2008, pp. 688-692, May 2008.

[19] P. Svedman, S. K. Wilson, and L. J. Cimini, "Opportunistic beamforming and scheduling for OFDMA systems," IEEE Transactions on Communications, vol. 55, no. 5, pp. 941-952, 2007.

[20] J. Choi and J. Ha, "Orthogonal beamforming for overlay mode of OFDMA-based rural broadband wireless access," in Proceedings of the 2012 IEEE Wireless Communications and Networking Conference, WCNC 2012, pp. 508-512, April 2012.

[21] M. Schwartz, W. Bennett, and S. Stein, Communication Systems and Techniques, McGraw-Hill, New York, NY, USA, 1966.

[22] Z. Wang and G. B. Giannakis, "A simple and general parameterization quantifying performance in fading channels," IEEE Transactions on Communications, vol. 51, no. 8, pp. 1389-1398, 2003. 

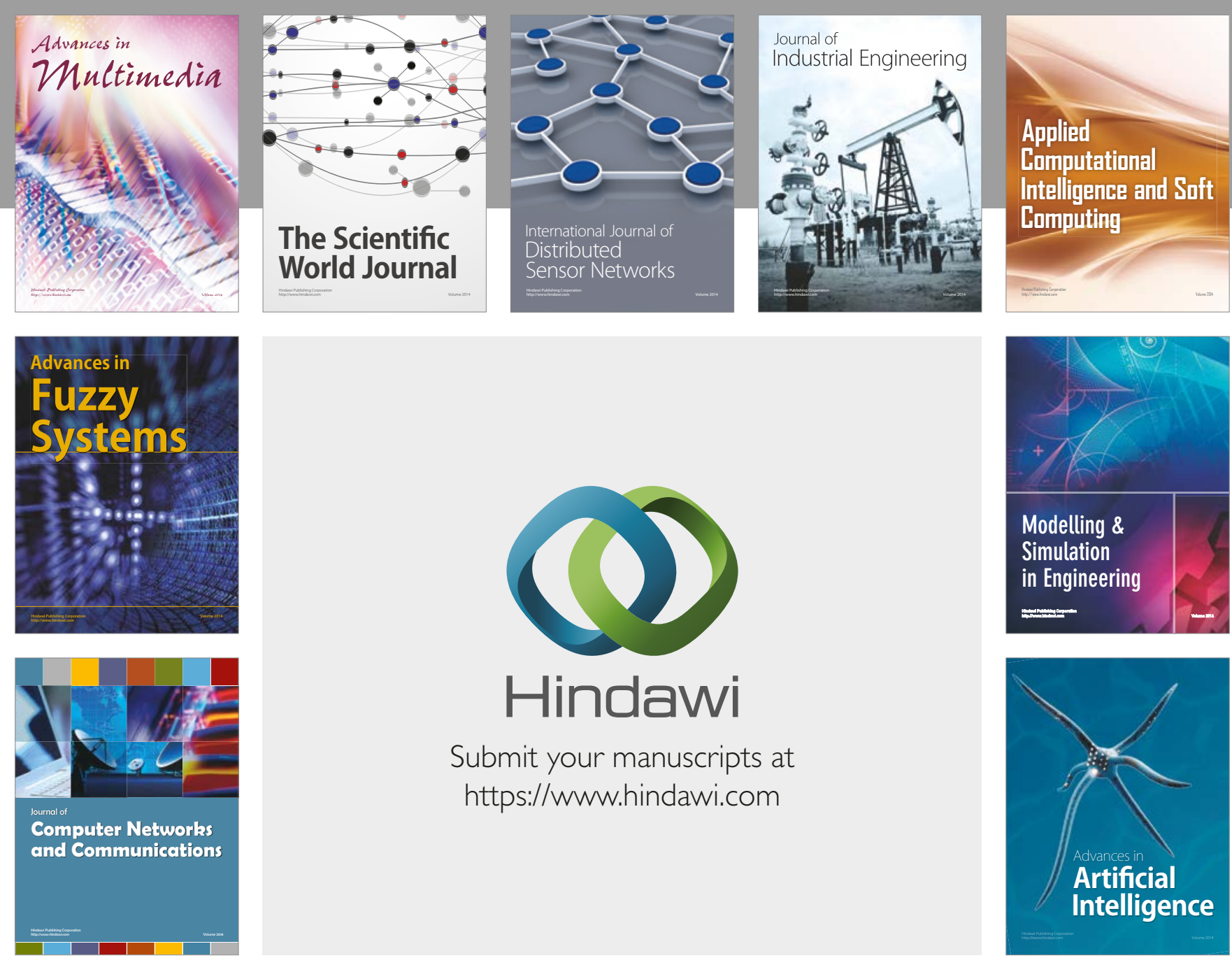

\section{Hindawi}

Submit your manuscripts at

https://www.hindawi.com
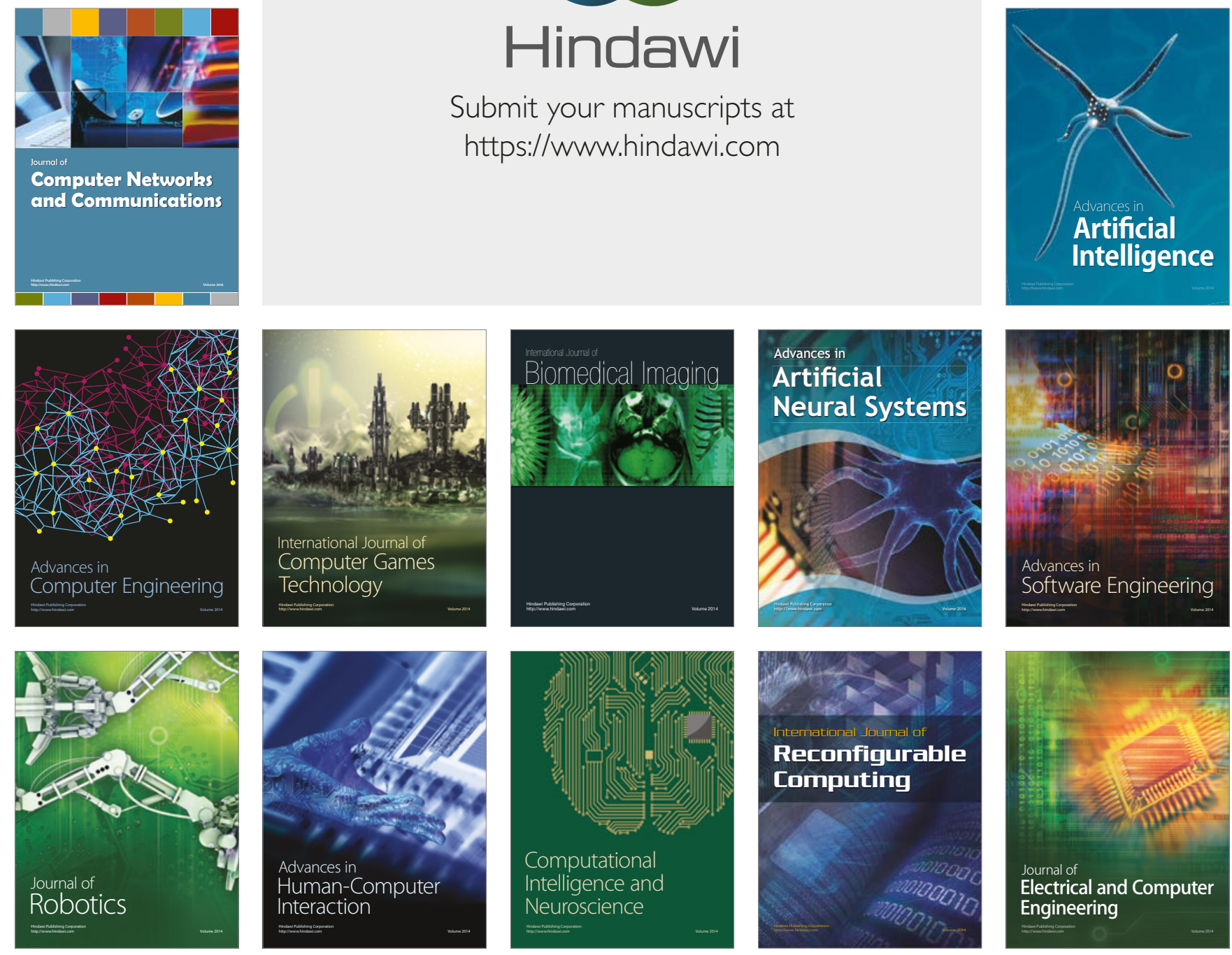